\title{
Nonequilibrium Equation of State in Suspensions of Active Colloids
}

\author{
Félix Ginot, ${ }^{1}$ Isaac Theurkauff, ${ }^{1}$ Demian Levis, ${ }^{2}$ Christophe Ybert, ${ }^{1}$ Lydéric Bocquet, ${ }^{1}$ \\ Ludovic Berthier, ${ }^{2}$ and Cécile Cottin-Bizonne ${ }^{1, *}$ \\ ${ }^{1}$ Institut Lumière Matière, CNRS UMR 5306, Université Claude Bernard Lyon 1, Université de Lyon, \\ Lyon, 69622 Villeurbanne Cedex, France \\ ${ }^{2}$ Laboratoire Charles Coulomb, CNRS UMR 5221, Université Montpellier 2, 34095 Montpellier, France
}

(Received 4 September 2014; published 23 January 2015)

\begin{abstract}
Active colloids constitute a novel class of materials composed of colloidal-scale particles locally converting chemical energy into motility, mimicking micro-organisms. Evolving far from equilibrium, these systems display structural organizations and dynamical properties distinct from thermalized colloidal assemblies. Harvesting the potential of this new class of systems requires the development of a conceptual framework to describe these intrinsically nonequilibrium systems. We use sedimentation experiments to probe the nonequilibrium equation of state of a bidimensional assembly of active Janus microspheres and conduct computer simulations of a model of self-propelled hard disks. Self-propulsion profoundly affects the equation of state, but these changes can be rationalized using equilibrium concepts. We show that active colloids behave, in the dilute limit, as an ideal gas with an activity-dependent effective temperature. At finite density, increasing the activity is similar to increasing adhesion between equilibrium particles. We quantify this effective adhesion and obtain a unique scaling law relating activity and effective adhesion in both experiments and simulations. Our results provide a new and efficient way to understand the emergence of novel phases of matter in active colloidal suspensions.
\end{abstract}

DOI: 10.1103/PhysRevX.5.011004

\section{INTRODUCTION}

Because they can be viewed as large atoms, colloids have been used to perform detailed explorations of equilibrium materials. Experimental studies of colloidal systems have largely contributed to the development of accurate statistical mechanics descriptions of classical states of matter $[1,2]$. Recently, "active colloidal matter" has emerged as a novel class of colloidal systems that is currently under intense scrutiny. Active colloids evolve far from equilibrium because they are self-propelled, motorized, or motile objects, for which active forces compete with interparticle interactions and thermal fluctuations. This broad class of systems encompasses bacterial colonies, epithelial tissues, and specifically engineered, or "synthetic," colloidal systems [3-5]. Progress in particle synthesis and tracking capability are such that frontier research in this field has shifted from single-particle studies to the understanding of physical properties of bulk active materials. Such large assemblies raise a number of basic physical challenges, from elucidation of two-body interactions to emerging many-body physics. In an effort to understand the various

*cecile.cottin-bizonne@univ-lyon1.fr

Published by the American Physical Society under the terms of the Creative Commons Attribution 3.0 License. Further distribution of this work must maintain attribution to the author(s) and the published article's title, journal citation, and DOI.
Subject Areas: Soft Matter, Statistical Physics

phases of matter emerging in active colloids, we study how self-propulsion affects the colloidal equation of state and provide a microscopic interpretation of our results.

Experimentally, active colloidal systems have been mostly characterized so far through detailed single-particle studies [6,7] in the dilute gas limit, where particles only interact with the solvent. In this regime, a mapping from nonequilibrium active systems to equilibrium passive ones was established in the presence of a gravitational field, where activity renormalizes the value of the effective temperature [8,9]. Theoretical analysis shows that this experimental result is not trivial, as the existence of a mapping to an effective "hot" ideal gas might break down for different geometries $[9,10]$ and also sensitively depends on the detailed modeling of self-propulsion [10,11].

Much less is understood at finite densities when particle interactions and many-body effects cannot be neglected. Several new phases of active matter have been observed experimentally in synthetic self-propelled colloids [12-14], from active cluster phases at relatively low density to gel-like solids and phase-separated systems at larger density, suggesting that active colloids tend to aggregate when selfpropulsion is increased. These observations have triggered a number of theoretical studies [15], which have suggested several physical mechanisms for particle aggregation. In particular, particles may aggregate in regions of large density because self-propulsion is hindered by steric effects [16-19]. Clustering is also observed when more complex coupling between motility, density, and reactant diffusion $[11,20,21]$ 
are taken into account. Motility-induced adhesion can be strong enough to induce a nonequilibrium phase separation between a dense fluid and a dilute gas, as observed in some recent studies, but complete phase separation does not always occur in simulations [19] or experiments [12,13] on active systems.

Here, we characterize the phase behavior and equation of state of a system of active colloids and of a model of self-propelled hard disks, extending to nonequilibrium suspensions the classical sedimentation studies pioneered by Perrin [22]. While theory and simulations have recently started to explore the equation of state of simple models of active particles [23-25], there is, so far, no corresponding experimental investigation of the pressure-density relation in active particle systems. We find that activity modifies the equation of state in a way that can be described by the introduction of both an effective temperature for the dilute system and an effective adhesive interaction at finite density. From the known equation of state for adhesive disks at equilibrium, we extract an effective adhesion between active particles and find a unique scaling law relating activity to self-propulsion in both experiments and simulations.

\section{SEDIMENTATION EXPERIMENTS IN NONEQUILIBRIUM ACTIVE SUSPENSIONS}

Sedimentation is a simple yet powerful tool to study colloidal suspensions because it allows a continuous exploration of the phase behavior of the system without finetuning of the volume fraction $[22,26]$. This technique has been used to explore phase diagrams and equations of state for several types of suspensions [27-32]. Sedimentation allows us to extend previous work on active colloids performed in the dilute regime [8] and at low density [12], to a much broader range of densities.

Experimentally, we study colloidal particles that are self-propelled through phoretic effects. We use gold Janus microspheres with one half covered with platinum. When immersed in a hydrogen-peroxide bath, the colloids convert chemical energy into active motion. The average radius obtained from scanning-electron-microscopy measurements is $R=1.1 \pm 0.1 \mu \mathrm{m}$, but image analysis at large density indicates an effective radius between 1.34 and $1.44 \mu \mathrm{m}$, decreasing slightly with activity. In the following, we assume that $R$ is constant, with the value given by the scanning-electron-microscopy measurements. Because of gravity, the Janus colloids (mass density $\rho \sim 11 \mathrm{~g} \mathrm{~cm}^{-3}$ ) form a bidimensional monolayer at the bottom of the observation chamber. We tilt the system by a small angle $\theta \sim 10^{-3}$ rad along the $z$ direction, as shown in the inset of Fig. 1(a), so that the gravity field felt by the particles is reduced to $g \sin \theta$. To study the effect of activity on the sedimentation, we measure the density profiles in the $z$ direction for various mass concentrations in $\mathrm{H}_{2} \mathrm{O}_{2}$ from 0 to $10^{-2} \mathrm{w} / \mathrm{w}$. The hydrogen peroxide concentration controls the level of particle self-propulsion, which can be quantified by the translational Péclet number $P_{e}$, defined as $P_{e}=\left(R v / D_{0}\right)$, where $D_{0}$ is the diffusion coefficient of the colloids in the absence of activity and $v$ is the average velocity of free microswimmers (see Appendix A). Notice that the present active-particle system was previously studied only at a single density [12], and the experimental setup to study its sedimentation profiles differs from earlier experimental studies [8].

We also conduct simulations of sedimentation profiles within a simple model of self-propelled hard disks [19,33]; see Fig. 1(b). The model is simpler than the experiments in two key aspects. First, it uses a purely hard-core repulsion between particles. This potential is a reasonable choice because the pair interaction between the Janus colloids has not been characterized in any detail, and it avoids the introduction of multiple control parameters. Second, the phoretic mechanism behind self-propulsion is not simulated, and particle activity is implemented directly into Monte Carlo equations of motion [19], which generalize
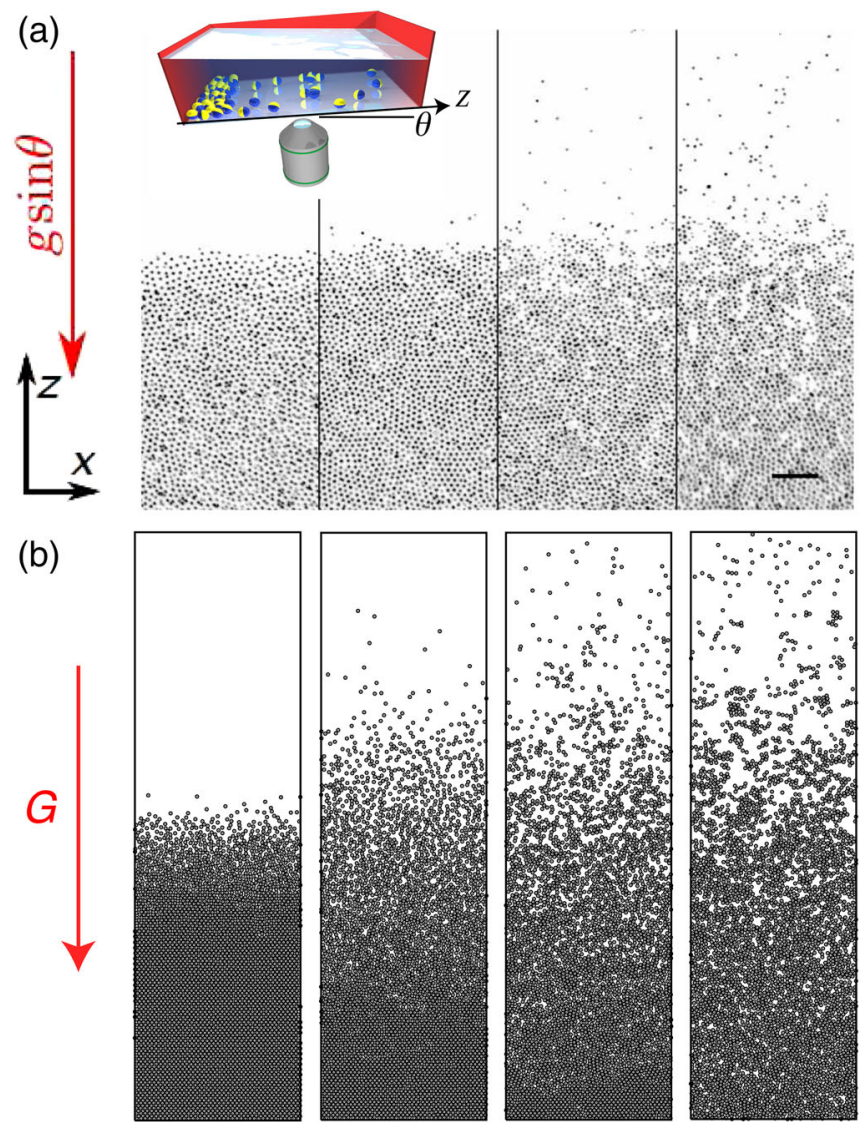

FIG. 1. Imaging sedimentation profiles. Snapshots of (a) phoretic gold-platinum Janus colloids and (b) self-propelled hard disks under gravity for activity increasing from left to right. (a) Four different activities are shown, corresponding to Péclet numbers $P_{e}=0$ (passive case), 8, 14, and 20 (from left to right). The inset shows a schematic view of the experimental setup. The scale bar is $20 \mu \mathrm{m}$. (b) Simulation snapshots for persistence times $\tau=0$ (passive disks), 1, 10, and 100 (from left to right). 
the algorithm used for Brownian hard disks to introduce self-propulsion. In the model, the activity is controlled by a single parameter, the persistence time $\tau$ (equivalent to a rotational Péclet number), determining the crossover time between ballistic and diffusive regimes in the dilute limit [19]. The intensity of the gravity force $G$ controls the sedimentation process (see Appendix B). The phase behavior and dynamics of the model without gravity have been carefully studied before $[19,33]$, but the equation of state has never been analyzed.

In Fig. 1, we show the sedimentation images for both the experimental system and the numerical model, for four different activity levels. The relevance of sedimentation studies is immediately clear, as the system continuously evolves from a very dilute suspension at the top to very dense configurations at the bottom. Therefore, a single experiment explores at once a broad range of densities for a given level of activity. Despite its simplicity, the numerical model reproduces qualitatively the various regimes observed for Janus colloids. For both systems, the passive case reveals equilibrium configurations from a dilute fluid to a dense homogeneous amorphous phase, while active systems exhibit much richer structures. The dilute gas spreads over larger altitudes, finite-size clusters are observed at moderate densities, gel-like configurations are found at larger densities, and dense, arrested phases exist at the bottom of the cell, as revealed by these images. These various phases have been carefully analyzed in the numerical model [19,33], but only the cluster phase was studied experimentally before [12]. In the following, we shall record and analyze quantitatively the equation of state of both systems over a range of densities where dynamics is not arrested and steady-state conditions can be achieved.

(a)

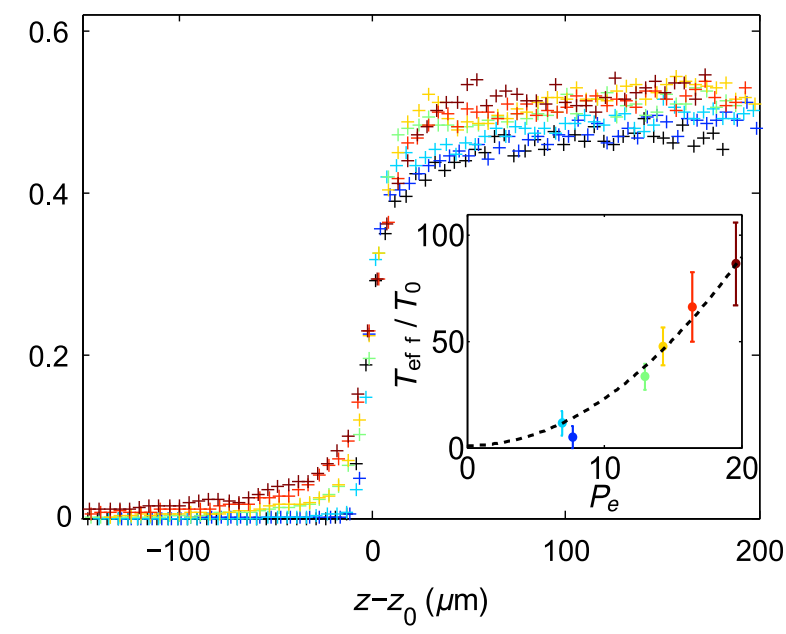

III. SEDIMENTATION PROFILES: DILUTE LIMIT AND EFFECTIVE TEMPERATURE

Our basic physical observable is the density profile $\phi(z)$ measured along the direction of the gravity from the images shown in Fig. 1 (see Appendix A). In Fig. 2(a), we show the density profiles obtained experimentally for different activities, which confirms the large range of densities explored in each experiment. We focus in Fig. 2(b) on the dilute phase at small density (large $z$ ) and observe that for $\phi \lesssim 0.05$, the density evolves exponentially with the position with a decay rate evolving continuously with the activity. We have obtained similar exponential profiles in the simulations.

The experimental results suggest that in the dilute limit, the system behaves as an ideal gas with an activitydependent effective temperature $T_{\text {eff }}$, such that $\phi(z) \sim$ $\exp \left[-m g z \sin \theta /\left(k_{B} T_{\text {eff }}\right)\right]$, where $m$ is the mass of a colloid and $k_{B}$ the Boltzmann constant. From the linear fits shown in Fig. 2(b), we extract the value of $k_{B} T_{\text {eff }} /(m g \sin \theta)$ for various activities. These measurements are more precise for $P_{e}$ above 10, as the dilute phase extends over larger distances; $k_{B} T_{0} /(m g \sin \theta)$ is then evaluated from the evolution of $k_{B} T_{\text {eff }} /(m g \sin \theta)$ with $P_{e}$. Overall, our results in this regime agree with earlier work on dilute suspensions [8], and the theoretical expression $T_{\text {eff }} / T_{0}=1+\frac{2}{9} P e^{2}$ is recovered; see the inset of Fig. 2(a). Quantitatively, we observe that $T_{\text {eff }}$ increases from the thermal bath temperature $T_{\text {eff }} \approx T_{0} \approx 300 \mathrm{~K}$ for passive colloids (from which we determine the tilt angle $\theta \approx 8 \times 10^{-3} \pm 2 \times 10^{-3}$ ) to a maximum value of about $T_{\text {eff }} \approx 3 \times 10^{4} \mathrm{~K}$ for the most active system.

In the simulations, we find similarly that $\phi(z)$ decays exponentially with $z$. We have verified numerically that the dependence on the gravity field $G$ is given by

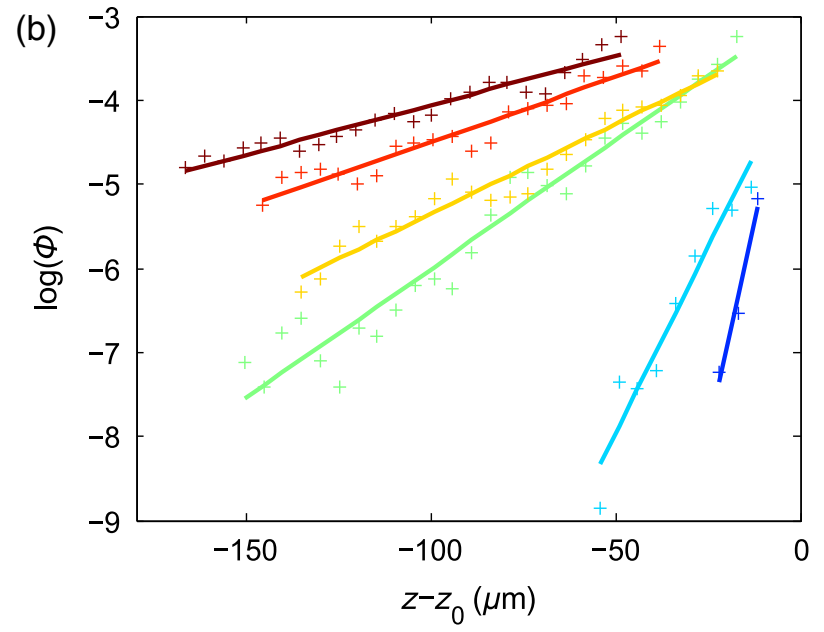

FIG. 2. Experimental density profiles. (a) Density profiles for various activities, arbitrarily shifted horizontally so that $z_{0}$ corresponds to $\phi=0.2$. Inset: Evolution of $T_{\text {eff }}$ as a function of the Péclet number, together with the theoretical expectation $T_{\text {eff }} / T_{0}=1+\frac{2}{9} P e^{2}$. (b) Zoom-in on the dilute phase $\phi \ll 1$ using a semilog scale, with the associated exponential fit, from which we extract the value of the effective temperature. 
$\phi(z) \sim \exp \left[-z G /\left(k_{B} T_{\text {eff }}\right)\right]$, which defines an effective temperature. Exponential decay is obeyed over about four decades for $\phi \in\left[10^{-6}, 10^{-2}\right]$. The lower bound stems from statistical accuracy, but the upper bound emerges because profiles become nonexponential when density is large enough for many-body interactions to play a role. Numerically, $T_{\text {eff }}$ is directly proportional to the persistence time of the self-propulsion $T_{\text {eff }} \propto \tau$. Therefore, selfpropelled hard disks under gravity also behave at low density $\phi \ll 10^{-2}$ as an ideal gas with an effective temperature different from the bath temperature $T_{\text {eff }}>T_{0}$.

To reinforce this view, we perform additional simulations in the absence of gravity, where we measure in the dilute limit $\phi \rightarrow 0$ both the self-diffusion coefficient $D_{s}$ (from the long-time limit of the mean-squared displacement) and the mobility $\mu$ (measured from the long-time limit of the response to a constant force in the linear response regime). The diffusion constant scales with the persistence time $D_{s} \propto \tau$, as expected [19], and we find that the mobility does
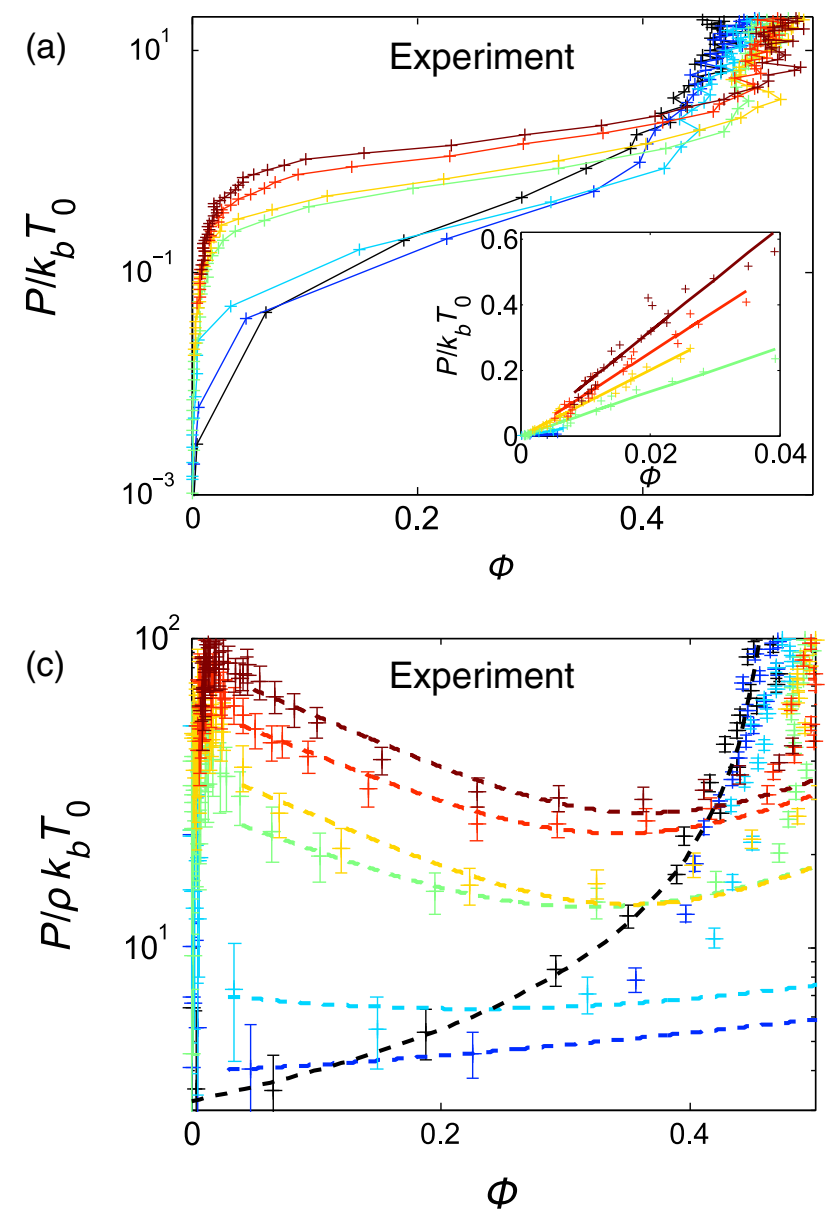

not scale with $\tau$, such that $D_{s} / \mu \propto \tau$. Our simulations indicate that the equality

$$
k_{B} T_{\text {eff }}=D_{s} / \mu
$$

holds quantitatively, within our statistical accuracy. Equation (1) states that the same effective temperature controls the sedimentation profiles and the (effective) Einstein relation between diffusion and mobility. Hence, it is justified to describe self-propelled particles as an "effective ideal gas." These conclusions are far from trivial, as they do not hold for all types of active particles (run-andtumble bacteria being a relevant counterexample [9]), while the mapping to an ideal gas may break down in more complex geometries, even for self-propelled particles [10]. Finally, note that $T_{\text {eff }}$ is a single-particle quantity, which is conceptually distinct from the (collective) effective temperature emerging in dense glassy regimes [34].
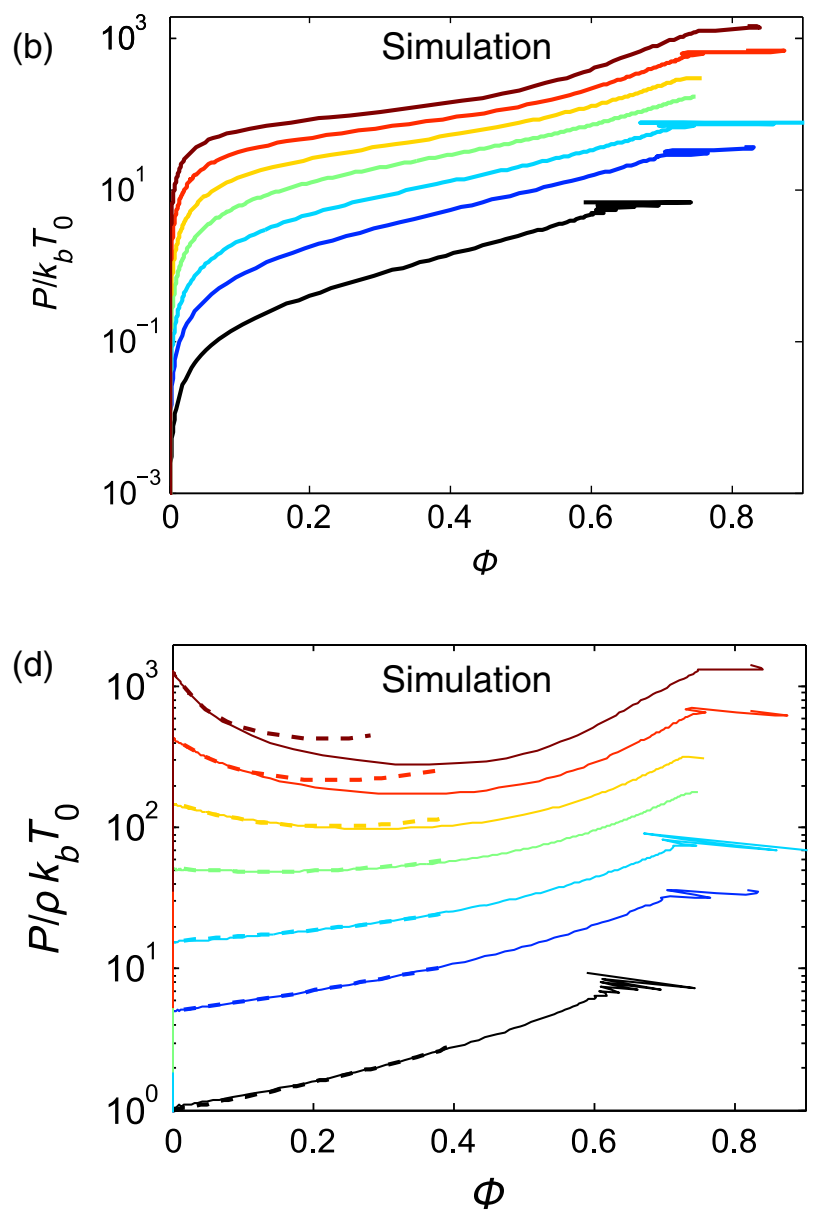

FIG. 3. Equation of state and compressibility factor. Equation of state $P /\left(k_{B} T_{0}\right)$ versus linear density $\phi$ for (a) experiment and (b) simulations. In (a), the inset zooms in on the dilute phase, where lines represent the best linear fit from which we recover $T_{\text {eff }}$. (c) Compressibility factor versus $\phi$ for experiment. Black points represent the passive case; the dashed black curve represents the empirical equation of state for hard disks. Dashed colored lines are obtained from Eq. (3). (d) Compressibility factor versus $\phi$ for simulations with dashed lines obtained from the Percus-Yevick closure for the two-dimensional Baxter model. Experiments: $T_{\text {eff }} / T_{0}=1,5,15,34,47,62,87$ (bottom to top). Simulations: $\tau=0,1,3,10,30,100,300,1000$ (bottom to top). 


\section{NONEQUILIBRIUM EQUATION OF STATE IN ACTIVE SUSPENSIONS}

Provided we measure density profiles in steady-state conditions, and using the sole assumption of mechanical equilibrium [27], we can convert the measured profiles into a pressure measurement $P(z)=\Pi(z)-\Pi_{0}$, where $\Pi_{0}$ is the pressure at the top of the observation cell and $\Pi(z)=\left(m g \sin \theta / \pi R^{2}\right) \int_{z}^{L} d z^{\prime} \phi\left(z^{\prime}\right)$. We can then represent the parametric evolution of $P(z)$ with $\phi(z)$ by varying $z$, which gives direct access to the nonequilibrium equation of state $P(\phi)$. To our knowledge, there is no previous experimental report of such quantities, which have only very recently been discussed in theoretical work [23-25]. In Figs. 3(a) and 3(b), we present the outcome of this analysis and show the evolution of the normalized osmotic pressure $P /\left(k_{B} T_{0}\right)$ for various activities in experiments and simulations. In Figs. 3(c) and 3(d), we replot the same set of data to examine how the compressibility factor $Z=P /\left(\rho k_{B} T_{0}\right)$, with $\rho$ the number density, depends on density $\phi$. This second representation offers a finer perspective on the nonequilibrium equation of state, as the ideal gas behavior at low $\phi$ is scaled out.

In the dilute regime, we recover the "effective" ideal gas behavior $P=\rho k_{B} T_{\text {eff }}$, which directly follows from integration of the exponential profiles of Fig. 2. This linear dependence of $P$ with $\phi$ is more carefully examined in Figs. 3(c) and 3(d), as it translates into a finite value for $Z(\phi \rightarrow 0)$, namely, $Z(\phi) \rightarrow T_{\text {eff }} / T_{0}$, as observed. The data in Figs. 3(c) and 3(d) thus provide a simple, direct measurement of the effective temperature in active suspensions.

More interesting is the behavior at finite density, which has not been explored experimentally before. Both experimental and numerical data indicate that the functional form of the equation of state changes continuously as the activity increases, in a way that cannot be uniquely accounted for by the introduction of the effective temperature. This is not surprising, as the images in Fig. 1 show that the structure of the system at finite density changes dramatically with activity. To confirm this change, we first analyze the data for passive systems. In that case, the equation of state can be well described by an empirical equation of state for hard disks [35,36]. The agreement with hard disks is not guaranteed in experiments because it is not obvious that (passive) Janus colloids uniquely interact with hard-core repulsion, but this seems to be a good approximation. In the simulation [black curves in Fig. 3(d)], we show that the data for passive disks agree very well with the equilibrium Percus-Yevick equation of state. This agreement is expected because the system becomes for $\tau=0$ an equilibrium fluid of hard disks.

When activity increases, experiments and simulations can no longer be described by the equilibrium hard-disk equation of state. Qualitatively, the compressibility factor $Z(\phi)$ increases more weakly with $\phi$ for moderately active particles than for the passive system, and we observe that
$Z(\phi)$ even decreases with $\phi$ for more active systems at low $\phi$, so that $Z(\phi)$ actually becomes a nonmonotonic function of $\phi$ for large activity [23-25]. In equilibrium systems, such a behavior represents a direct signature of adhesive interactions [31]. This behavior suggests that the strong clustering observed in Fig. 1 in active-particle systems directly impacts the equation of state, which takes a form reminiscent of equilibrium colloidal systems with attractive interactions.

\section{DETERMINATION OF EFFECTIVE ADHESION INDUCED BY SELF-PROPULSION}

The idea that self-propulsion provides a mechanism for inducing effective attractive forces in purely repulsive systems of active particles has recently emerged [14,16, $25,37]$. Because we have direct access to the equation of state in our systems of active particles, we are in a unique position to study the similarity between the equation of state of nonequilibrium active particles and equilibrium adhesive disks. If successful, we can then quantify the strength of the effective adhesion between particles, which is induced by the self-propulsion mechanism.

To this end, we compare the equation of state obtained for active particles to the equilibrium equation of state of a system of adhesive particles. We have analyzed the equation of state of the Baxter model of adhesive particles in two dimensions [38]. This model is defined by a squarewell potential with a hard-core repulsion for $r<\sigma$ and a short-range attraction of range $\sigma+\delta$ and depth

$$
V(\sigma<r<\sigma+\delta)=-k_{B} T \ln \left(\frac{\sigma+\delta}{4 \delta} A\right),
$$

where $A$ is a nondimensional number quantifying the adhesion strength and $V(r>\sigma+\delta)=0$. The model is defined such that the adhesive limit can smoothly be taken, where $\delta \rightarrow 0, V\left(r=\sigma^{+}\right) \rightarrow \infty$, but the second virial coefficient remains finite and is uniquely controlled by the dimensionless adhesion parameter $A[38,39]$. The purely repulsive hard-disk limit is recovered with $A \rightarrow 0$. For this model, the first two virial coefficients are known analytically [40]:

$$
Z=\frac{P}{\rho k_{B} T}=1+b_{1} \phi+b_{2} \phi^{2}+\mathcal{O}\left(\phi^{3}\right),
$$

with $b_{1}=2-A$ and $b_{2}=\frac{25}{8}-\frac{25}{8} A+\frac{4}{3} A^{2}-0.122 A^{3}$. When $A$ increases, the compressibility factor $Z$ in Eq. (3) changes from the monotonic hard-disk behavior to a nonmonotonic density dependence for $A>2$ as the initial slope given by $b_{1}$ then becomes negative.

The above expression provides a reasonable description of the experimental data, provided we carefully adjust $A$ for each activity. This adjustment provides a direct estimate of the "effective adhesion" induced by the self-propulsion, 


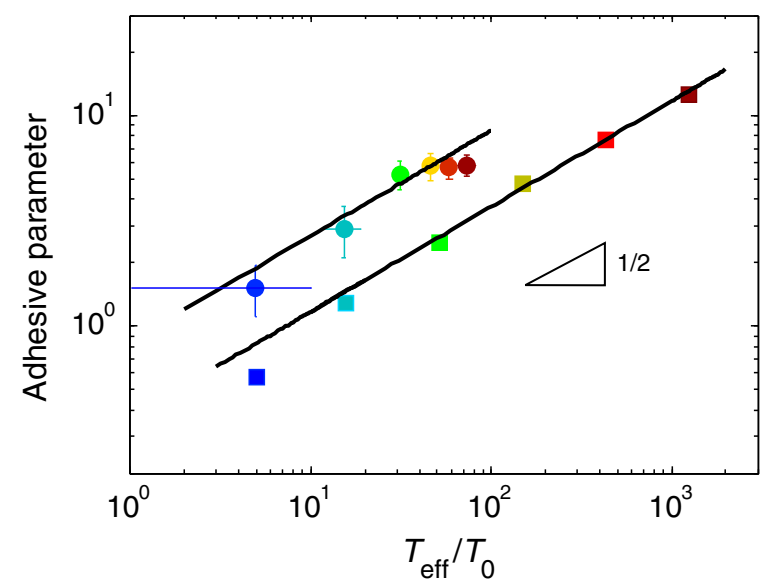

FIG. 4. Quantifying motility-induced adhesion. Evolution of adhesive parameter $A$ obtained from the equation of state with the effective temperature obtained in the dilute limit for both experiments (circles) and simulations (squares). The lines correspond to the scaling law $A \sim \sqrt{T_{\text {eff }} / T_{0}}$, with slightly different prefactors for simulations and experiments.

which thus quantifies the nonequilibrium clustering of selfpropelled particles using a concept drawn from equilibrium physics. In practice, we fit the experimental compressibility factor at intermediate densities $(0.04<\phi<0.4)$ with the expression $Z=\alpha\left[1+b_{1} \phi+b_{2} \phi^{2}+\mathcal{O}\left(\phi^{3}\right)\right]$, adjusting both the effective adhesion $A$ and a prefactor $\alpha$, which accounts for the effective temperature. Within error bars, we obtain $\alpha \approx T_{\text {eff }} / T_{0}$, confirming the robustness of the analysis. We have also verified that the uncertainty on the determination of $\phi$ (due to the uncertainty in particle diameter) has a negligible impact on the measured $A$ values. As shown in Fig. 4, we find that the effective adhesion $A$ increases with self-propulsion.

We carry out a similar analysis for the compressibility factors obtained numerically for self-propelled hard disks. Because the numerical data are statistically accurate over a broad density range, we solve the full Percus-Yevick closure relation to obtain the equation of state of the bidimensional Baxter model and adjust $A$ to obtain the best agreement between self-propelled and adhesive disks, as shown in Fig. 3(d). As observed in the experiments, we again obtain an adhesion $A$ that increases with selfpropulsion; see Fig. 4.

To analyze the evolution of the effective adhesion with activity, it is useful to represent $A$ as a function of $T_{\text {eff }} / T_{0}$. This representation is convenient because it only involves dimensionless quantities, and $T_{\text {eff }} / T_{0}$ efficiently quantifies self-propulsion with no reference to its detailed physical origin. ( $T_{\text {eff }}$ is proportional to the diffusion constant of noninteracting particles.) Remarkably, we observe a similar scaling relation for both experiments and simulations between adhesion and effective temperature, namely, $A \sim \sqrt{T_{\text {eff }} / T_{0}}$.

\section{PHYSICAL DISCUSSION}

The scaling law relating $A$ and $T_{\text {eff }}$ suggests that selfpropelled disks at finite density do not simply behave as a "hot" suspension of hard disks but rather as a hot suspension of adhesive disks with a simple relation between adhesion and effective temperature. A plausible scaling argument for the emergence of such a scaling is to compare the contact duration for active particles, controlled by the rotational Péclet number (or persistence time), to a bonding time in the context of the Baxter model $t_{\text {bond }} \sim \exp \left(-V / k_{B} T\right) \sim A$. This simple argument suggests, however, an incorrect proportionality between $A$ and $T_{\text {eff }}$, which demonstrates that the scaling of the active adhesion follows from a more complex interplay between bonding time and structural evolution induced by the self-propulsion.

To rationalize the observed scaling, we have compared quantitatively the structure obtained with self-propelled disks at finite density to computer simulations of the equilibrium Baxter model of adhesive disks [40]. In particular, we find that the degree of clustering (quantified by the average cluster size) is the same in both models when $A$ and $\sqrt{T_{\text {eff }}}$ increase proportionally, which gives further support to the results found in Fig. 4. In previous work, it was shown that the average cluster size increases as $\sqrt{T_{\text {eff }}}$ in our numerical model [19]. This $\sqrt{T_{\text {eff }}}$ scaling law is actually captured by a kinetic model of reversible aggregation [19], which assumes that clusters result from a competition between particle aggregation (when a selfpropelled particle collides with an existing cluster) and escape from the cluster surface (when the direction of the self-propulsion for a surface particle becomes oriented toward the exterior). While this physical model is in exactly the spirit as the above scaling argument based on a bonding time scale, the kinetic model is able to capture the manybody physics of particle exchanges between dynamic clusters responsible for the observed connection between adhesion and activity.

The results in Fig. 4 indicate that, while simulations and experiments obey the same scaling, the experimental adhesive parameter is systematically larger. This difference suggests that a second mechanism could be at work in experiments. Following Refs. [12,21], we may attribute it to the chemical (phoretic) interactions between colloids. The fuel-powering phoretic motion is depleted around the colloids, which may induce a diffusiophoretic attraction between colloids. From the force balance between colloids, this interaction can be quantified in terms of an attraction energy that is directly proportional to the chemical consumption rate and, therefore, to the self-propelled velocity [12]. Altogether, this mechanism suggests an attraction energy scaling like $A \sim P_{e} \sim \sqrt{T_{\text {eff }}}$, as observed. Our experiments and simulations, therefore, point to an additive combination of kinetic and phoretic contributions to the experimentally observed effective attraction. 
Our approach provides a quantitative way to account for the clustering commonly observed in self-propelled particle systems, as it suggests that active particles effectively behave as an equilibrium system of hot adhesive particles, at least in the regime explored experimentally in this work. In the experiments, we can notice that the effective adhesion $A$ seems to saturate with the effective temperature; see Fig. 4. This saturation could be linked to the fact that in our experiments, we do not observe a macroscopic phase separation. In adhesive spheres, phase separation occurs for larger adhesion values $A>10$ [38], while clustering is observed [40] for adhesion values corresponding to our experiments. Finally, it is remarkable that despite the complex mechanisms responsible for self-propulsion at the microscale, the experimental equation of state is very well reproduced in a very simple model of self-propelled hard disks. This result suggests that Janus active colloids represent an interesting experimental model system to study the statistical physics of active colloidal matter.

\section{ACKNOWLEDGMENTS}

We thank T. Biben, H. Löwen, and G. Szamel for fruitful discussions, B. Abécassis for the synthesis of the gold colloids, Institut des Nanotechnologies de Lyon for access to its clean room facility, and A. Ikeda for his help in the numerical integration of the Percus-Yevick equation. The research leading to these results has received funding from the European Research Council under the European Union's Seventh Framework Programme (FP7/2007-2013)/European Research Council Grant Agreement No. 306845.

F. G. and I. T. contributed equally to this work.

\section{APPENDIX A: EXPERIMENTS ON JANUS ACTIVE COLLOIDS}

We typically study the sedimentation of about $10^{6}$ Janus colloids. After each increase in peroxide, we wait for $5 \mathrm{~min}$ to reach a stationary state inside the cell. The chamber depth is about $6 \mathrm{~mm}$ for a total volume in $\mathrm{H}_{2} \mathrm{O}_{2}$ of $300 \mu \mathrm{L}$ so that there is sufficient fuel for the whole duration of the experiment (see the movie in the Supplemental Material [41]). In those conditions, particles remain active during several hours. The Janus colloids behave as small batteries, disproportionating the peroxide into water and dissolved dioxygen [42]. These chemical reactions create electrochemical gradients around the colloid that lead to motion at $v \sim 2 \mu \mathrm{m} \mathrm{s}^{-1}$, depending on the concentration in peroxide. The Péclet number can be used to describe the activity of the particles and is defined as $P_{e}=(R v / D)$, where $D=0.21 \pm 0.05 \mu \mathrm{m}^{2} \mathrm{~s}^{-1}$ is the diffusion coefficient of the colloids in the absence of activity and $v$ is the average velocity of free microswimmers, defined as colloids without neighbors closer than $6 \mu \mathrm{m}$. Our basic observable for the analysis is the density profile $\phi(z)$. In the experimental system, we perform a very basic coarse-graining operation on pictures, counting the number of colloids $n(x, z)$ in square boxes of size $l \sim 5 \mu \mathrm{m}$. Then, we compute the number density $\rho(z)=(1 / L) \int_{0}^{L}\left(n(x, z) / l^{2}\right) d x$ and the density profile $\phi(z)=\left(\pi R^{2} / L\right) \int_{0}^{L}\left(n(x, z) / l^{2}\right) d x=$ $\pi R^{2} \rho(z)$, where $L \times L=350 \times 350 \mu \mathrm{m}^{2}$ is the size of the observation area. For each peroxide concentration, 2000 4-Mpx frames are taken at $20 \mathrm{~Hz}$ using a Baumer HXC40 camera mounted on a Leica DMI 4000B microscope and a custom-made external dark-field lightning ring. Particle trajectories are reconstructed using ordinary tracking algorithms. The error bars are determined from the standard deviation of the fluctuations of independent measurements of the density obtained by coarse graining the sample in 60 independent boxes along the $x$ axis. Moreover, as we are in a stationary state, this statistics is completed by ten independent realizations.

\section{APPENDIX B: SIMULATIONS OF SELF- PROPELLED HARD DISKS}

To study sedimentation profiles, we introduce a hard wall at position $z=0$ while keeping periodic boundary conditions in the $x$ direction. We introduce gravity as a force $\vec{G}$ acting along the $z$ direction [see Fig. 1(b)], so that a particle displacement $\Delta z$ along the $z$ direction is accepted with the Metropolis acceptance rule $\min [1, \exp (-\beta \Delta z G)]$, where $\beta=\left(k_{B} T\right)^{-1}$. Under the influence of gravity, particles accumulate at the bottom of the simulation cell, whose height is taken large enough that the top wall does not influence the results. We report results for simulations with $N=6144$ disks in a box of width $W=40 \sigma$, where $\sigma$ is the disk diameter. We have performed tests with $N=1024,3072$, and 12288 disks to establish that the present results are not influenced by finite-size effects. For each value of $\tau$ controlling the self-propulsion, we perform simulations for several values of the gravity field $G$ and check that the results presented below for the equations of state obtained in steady-state conditions do not depend on the chosen value. The density profile $\phi(z)$ is obtained by performing very long simulations of the steady-state profiles and performing a time average for each pair $(\tau, G)$.

[1] P. N. Pusey and W. van Megen, Phase Behavior of Concentrated Suspensions of Nearly Hard Colloidal Spheres, Nature (London) 320, 340 (1986).

[2] J.-P. Hansen and I. R. McDonald, Theory of Simple Liquids (Elsevier, New York, 2005).

[3] P. Ball, Colloids Get Active, Nat. Mater. 12, 696 (2013).

[4] W. C. K. Poon, in Physics of Complex Colloid, Proceedings of the International School of Physics "Enrico Fermi," Course CLXXXIV, edited by C. Bechinger, F. Sciortino, and P. Ziherl (IOS, Amsterdam, 2013), p. 317-386.

[5] S. Ramaswamy, The Mechanics and Statistics of Active Matter, Annu. Rev. Condens. Matter Phys. 1, 323 (2010). 
[6] W. F. Paxton, A. Sen, and T. E. Malhouk, Motility of Catalytic Nanoparticles through Self-Generated Forces, Chem. Eur. J. 11, 6462 (2005).

[7] J. R. Howse, R. A. L. Jones, A. J. Ryan, T. Gough, R. Vafabakhsh, and R. Golestanian, Self-Motile Colloidal Particles: From Directed Propulsion to Random Walk, Phys. Rev. Lett. 99, 048102 (2007).

[8] J. Palacci, C. Cottin-Bizonne, C. Ybert, and L. Bocquet, Sedimentation and Effective Temperature of Active Colloidal Suspensions, Phys. Rev. Lett. 105, 088304 (2010).

[9] J. Tailleur and M. E. Cates, Sedimentation, Trapping, and Rectification of Dilute Bacteria, Europhys. Lett. 86, 60002 (2009).

[10] G. Szamel, Self-Propelled Particle in an External Potential: Existence of an Effective Temperature, Phys. Rev. E 90, 012111 (2014).

[11] M. Enculescu and H. Stark, Active Colloidal Suspensions Exhibit Polar Order under Gravity, Phys. Rev. Lett. 107, 058301 (2011).

[12] I. Theurkauff, C. Cottin-Bizonne, J. Palacci, C. Ybert, and L. Bocquet, Dynamic Clustering in Active Colloidal Suspensions with Chemical Signaling, Phys. Rev. Lett. 108, 268303 (2012).

[13] J. Palacci, S. Sacanna, A. P. Steinberg, D. J. Pine, and P. M. Chaikin, Living Crystals of Light-Activated Colloidal Surfers, Science 339, 936 (2013).

[14] I. Buttinoni, J. Bialké, F. Kümmel, H. Löwen, C. Bechinger, and T. Speck, Dynamical Clustering and Phase Separation in Suspensions of Self-Propelled Colloidal Particles, Phys. Rev. Lett. 110, 238301 (2013).

[15] P. Ball, Particle Clustering Phenomena Inspire Multiple Explanations, Physics 6, 134 (2013).

[16] J. Tailleur and M.E. Cates, Statistical Mechanics of Interacting Run-and-Tumble Bacteria, Phys. Rev. Lett. 100, 218103 (2008).

[17] Y. Fily and M. C. Marchetti, Athermal Phase Separation of Self-Propelled Particles with No Alignment, Phys. Rev. Lett. 108, 235702 (2012).

[18] G. S. Redner, M. F. Hagan, and A. Baskaran, Structure and Dynamics of a Phase-Separating Active Colloidal Fluid, Phys. Rev. Lett. 110, 055701 (2013).

[19] D. Levis and L. Berthier, Clustering and Heterogeneous Dynamics in a Kinetic Monte-Carlo Model of SelfPropelled Hard Disks, Phys. Rev. E 89, 062301 (2014).

[20] B. M. Mognetti, A. Saric, S. Angloletti-Uberti, A. Cacciuto, C. Valeriani, and D. Frenkel, Living Clusters and Crystals from Low-Density Suspensions of Active Colloids, Phys. Rev. Lett. 111, 245702 (2013).

[21] O. Pohl and H. Stark, Dynamic Clustering and Chemotactic Collapse of Self-Phoretic Active Particles, Phys. Rev. Lett. 112, 238303 (2014).

[22] J. Perrin, Mouvement Brownien et Réalité Moléculaire, Ann. Chim. Phys. 18, 1 (1909).

[23] S. A. Mallory, A. Saric, C. Valeriani, and A. Cacciuto, Anomalous Thermomechanical Properties of a Self-Propelled Colloidal Fluid, Phys. Rev. E 89, 052303 (2014).
[24] X. Yang, L. Manning, and M. C. Marchetti, Aggregation and Segregation of Confined Active Particles, arXiv: 1403.0697.

[25] S. C. Takatori, W. Yan, and J. F. Brady, Swim Pressure: Stress Generation in Active Matter, Phys. Rev. Lett. 113, 028103 (2014).

[26] R. Piazza, Settled and Unsettled Issues in Particle Settling, Rep. Prog. Phys. 77, 056602 (2014).

[27] T. Biben, J.-P. Hansen, and J.-L. Barrat, Barometric Equilibrium as a Probe of the Equation of State of Colloidal Suspensions, J. Phys. Condens. Matter 4, L11 (1992).

[28] T. Biben, J.-P. Hansen, and J.-L. Barrat, Density Profiles of Concentrated Colloidal Suspensions in Sedimentation Equilibrium, J. Chem. Phys. 98, 7330 (1993).

[29] R. Piazza, T. Bellini, and V. Degiorgio, Equilibrium Sedimentation Profiles of Screened Charged Colloids: A Test of the Hard-Sphere Equation of State, Phys. Rev. Lett. 71, 4267 (1993).

[30] M. A. Rutgers, J. H. Dunsmuir, J.-Z. Xue, W. B. Russel, and P. M. Chaikin, Measurement of the Hard-Sphere Equation of State Using Screened Charged Polystyrene Colloids, Phys. Rev. B 53, 5043 (1996).

[31] S. Buzzaccaro, R. Rusconi, and R. Piazza, "Sticky” Hard Spheres: Equation of State, Phase Diagram, and Metastable Gels, Phys. Rev. Lett. 99, 098301 (2007).

[32] L. J. Daniels, T. K. Haxton, N. Xu, A. J. Liu, and D. J. Durian, Temperature-Pressure Scaling for Air-Fluidized Grains on Approaches to Point J, Phys. Rev. Lett. 108, 138001 (2012).

[33] L. Berthier, Nonequilibrium Glassy Dynamics of SelfPropelled Hard Disks, Phys. Rev. Lett. 112, 220602 (2014).

[34] L. Berthier and J. Kurchan, Nonequilibrium Glass Transitions in Driven and Active Matter, Nat. Phys. 9, 310 (2013).

[35] A. Santos, M. L. de Haro, and S. B. Yuste, An Accurate and Simple Equation of State for Hard Disks, J. Chem. Phys. 103, 4622 (1995).

[36] We use the following equation of state [35]: $Z(\phi)=(1-$ $\left.2 \phi+\left(2 \phi_{\max }-1 / \phi_{\max ^{2}}\right) \phi^{2}\right)^{-1}$, where we adjust $\phi_{\max }=0.47$, in order to account for the uncertainty on the particle size discussed in the main text.

[37] J. Bialké, H. Löwen, and T. Speck, Microscopic Theory for the Phase Separation of Self-Propelled Repulsive Disks, Europhys. Lett. 103, 30008 (2013).

[38] R. J. Baxter, Percus-Yevick Equation for Hard Spheres with Surface Adhesion, J. Chem. Phys. 49, 2770 (1968).

[39] M. G. Noro and D. Frenkel, Extended Corresponding-States Behavior for Particles with Variable Range Attractions, J. Chem. Phys. 113, 2941 (2000).

[40] A. J. Post and E. D. Glandt, Cluster Concentrations and Virial Coefficients for Adhesive Particles, J. Chem. Phys. 84, 4585 (1986).

[41] See Supplemental Material at http://link.aps.org/ supplemental/10.1103/PhysRevX.5.011004 for movies of sedimentation for increasing activities.

[42] J. L. Moran and J. D. Posner, Electrokinetic Locomotion due to Reaction-Induced Charge Auto-electrophoresis, J. Fluid Mech. 680, 31 (2011). 